standards we operate a weekly attending consultant system with resident on calls until 9pm, with a focus on acutely unwell children including fulfilling standard of consultant review of acute admission within 14 hours of admission. We have increasingly realised that this model of care in insufficient to care effectively for patients with more complex medical and social health needs.

We are to piloting a new model of care to improve the care of Children with Complex Medical needs. This involves a second Ward Attending Consultant to focus on their care, supported by a new administrative role and Clinical nurse specialist (CNS) role.

Method The Second Attending Consultant of the week, instead of managing acute medical admissions focusses on these children and families supported by an administrator and the Complex CNS. The model moves away from the daily ward rounds needed by acute patients, with instead twice weekly reviews and a greater focus on coordinating effective Multidisciplinary meetings, addressing psychosocial needs and good communication with families. The changes are based on Qualitative feedback from Staff and Family. Regular evaluation of the effectiveness of the service change has been started alongside a narrative of barriers to change and complications to help inform next steps.

Results Monthly data is collected as indirect measures of improved communication between professionals and has improved from as baseline; time to first Multidisciplinary meeting, time Community teams informed of admission and discharge. Quantitative data regarding length of stay, A\&E attendance, hospital admissions and missed Community appointments due to inpatient stay has been collected as a baseline. Early data shows a reduction in length of stay, data collection for other parameters are planned in January 2020 alongside repeat staff and patient surveys.

Conclusion The paediatric complex care second attending model has enabled improved communication and reduced length of stay for patients with complex medical and social needs

\section{G508 A NATIONAL PICTURE OF CHALLENGES FACED BY PAEDIATRIC UNITS IN DISCHARGE OF CHILDREN WITH MEDICAL COMPLEXITY}

M Salama, RK Shanahan. Children with Medical Complexities Team, Birmingham Children's Hospital, Birmingham, UK

\subsection{6/archdischild-2020-rcpch.433}

Aim The prevalence of children with medical complexity (CMC) has increased in recent decades. CMC often are subject to increased lengths of stays (LOS). Discharge teams within UK paediatric services are more heterogenous as compared to counterpart adult services. Our aim was to explore how discharge for CMC children was facilitated in UK paediatric units.

Method We obtained a list of all UK Paediatric units from the Royal College of Paediatrics and Child Health. We contacted all the units with a freedom of information request for 8 questions. Questions 1-3 were to ascertain knowledge base of numbers of CMC or high LOS inpatients. Questions 4-5 were around non-medical barriers to discharge and Questions 6-8 involved exploration of how discharge planning was facilitated. A second contact was made to non-responders.
Results Of 186 units, 13 duplicate or inactive departments were eliminated. Following the 2 contacts we received a response rate of $86 \%$. Few units were able to reply to the specific question around our CMC definition. There was more clarity around LOS with $71 \%$ of units having $<5$ long stay patients. Of those who replied, just under half the units had patients with non-medical barriers to discharge. Housing, care and commissioning and social barriers were the most common. Tertiary centres were more likely to have non-medical barriers to discharge. Discharge was mostly coordinated by nurses. $8 \%$ of units had a discharge coordinator. There were links to charity support in a large proportion of cases with hospices being most common. Charities such as Wellchild, Roald Dahl and others were mentioned. The most commonly cited paperwork used by this group of children were advanced care plans and hospital passports.

Conclusion Our responses have demonstrated the similarity of challenges faced in supporting CMC and their discharge. CMC numbers are not routinely collected which may mean less visibility of the challenges that are faced. We are seeking to be part of a workstream to share good learning and drive service development for this growing and yet vulnerable group of patients.

\section{G509(P) AN EVALUATION OF 10 YEARS OF CLINICAL PROVISION FOR CHILDREN WITH MEDICALLY UNEXPLAINED SYMPTOMS IN A PAEDIATRIC PSYCHOLOGY SERVICE}

\footnotetext{
1,2S Robinson, ${ }^{1,3} \mathrm{~L}$ McGunnigle, ${ }^{1} \mathrm{~K}$ Golding, ${ }^{3} \mathrm{~L}$ Ah-Wan, ${ }^{1} \mathrm{G}$ Colville. ${ }^{1}$ Paediatric Psychology Service, St Georges University Hospital, London, UK; ${ }^{2}$ Children's Neurosciences, Evelina London Children's Hospital, London, UK; ${ }^{3}$ The School of Psychology, University of Surrey, Guildford, UK
}

\subsection{6/archdischild-2020-rcpch.434}

Aims Medically unexplained symptoms (MUS) are distressing somatic experiences that appear to be of physical origin, but are medically unexplained. Despite evidence from the adult literature that these cases are associated with high utilisation of health care resources, little is known about the impact on services of MUS in children. The aim of this study was to evaluate service use of children with MUS referred to a Paediatric Psychology Service (PPS) at an acute hospital, over a 10 year period, with the view to informing service developments for these patients.

Method Demographic, referral and clinical activity data were available for 268 patients with MUS and 4287 without, referred between 2007 and 2017.

Results Patients with MUS accounted for 4-8\% of referrals annually. Symptoms most commonly reported included unexplained pain, often in the abdomen (33\%), dizziness/fainting/ headaches (21\%), and functional neurological symptoms such as abnormal motor functioning (13\%) and non-epileptic attacks (12\%).

Children with MUS were significantly more likely to be female $\quad(\mathrm{MUS}=56 \%$, non-MUS $=49 \%, \mathrm{p}<0.01)$ and older (MUS=12.5 years, non-MUS=9.2 years, $\mathrm{p}<0.001$ ). In all, $54 \%$ of children with MUS also had a co-occurring medical diagnosis. Patients with MUS required significantly more outpatient clinical sessions than patients without MUS (mean: MUS $=10.7$, non-MUS $=5.4, \mathrm{p}<0.001$ ) and were also more likely to cancel appointments (MUS $=0.7 \%$, non-MUS $=0.4 \%$, $\mathrm{p}<0.01)$. Patient groups did not differ for duration of clinical input or whether clinical objectives were partially/wholly met. 